\title{
Immunoimaging of choroidal melanoma: assessment of its diagnostic accuracy and limitations in 101 cases
}

\author{
J Scheidler, G Leinsinger, C-M Kirsch, O F Scheiffarth, F H Stefani, K G Riedel
}

\begin{abstract}
Immunoscintigraphy (IS) was performed on 101 patients with space occupying intraocular lesions including choroidal melanomas (85), choroidal naevi (11), non-melanoma metastases (three), and other melanoma simulating lesions (two). Scintigraphic images with conventional and emission computer tomography techniques were obtained after the intravenous injection of ${ }^{99 m}$ Tc-labelled $F\left(a b^{\prime}\right)_{2}$ fragments of monoclonal antibody (MoAb) 225.28S directed against the high molecular weight-melanoma associated antigen (HMWMAA). Immunohistochemistry was performed on sections of four out of 10 melanomacontaining eyes to confirm MoAb binding. IS demonstrated positive scans in 66 out of 85 choroidal melanomas, offering a sensitivity of $78 \%$. Sensitivity was dependent on the lesion size. True negative results were obtained in $\mathbf{1 5}$ out of 16 non-melanoma lesions (specificity 94\%). False positive antibody accumulation was found in one patient with a post-traumatic subretinal haemorrhage. Immunohistochemistry demonstrated positive MoAb 225.28S binding in all melanoma sections. In summary IS offered substantial sensitivity and specificity in the differentiation of intraocular lesions, particularly choroidal melanomas, naevi, and metastases. In combination with other diagnostic procedures such as ultrasound echography and fluorescein angiography IS proved to be a valuable method in the diagnosis of choroidal melanoma.
\end{abstract}

(BrF Ophthalmol 1992; 76: 457-460)

At present a variety of diagnostic modalities such as ophthalmoscopy, ultrasound echography, transillumination, and fluorescein angiography are used in the differentiation of intraocular lesions. The combination of ophthalmoscopy, fluorescein angiography, and ultrasound echography provides the highest accuracy. ${ }^{.}$However difficulties still exist in distinguishing a small melanoma from a naevus, a haemangioma, and a metastasis. Echographic characteristics of malignancies are hard to establish when tumour thickness is low or not determinable.

Radioisotopes in the diagnosis of ocular melanoma were formerly extensively used in the ${ }^{32} \mathrm{P}$ test. Both, the necessity of a surgical procedure to place a probe onto the sclera for $\beta$ count measurement and some false positive results, due to the non-specific tracer accumulation, raised doubts of its clinical value. ${ }^{2}$ Another attempt was the administration of ${ }^{51} \mathrm{Cr}$ bleomycin, a non-specific oncophilic radiopharmaceutical. Sensitivity and specificity were reported to be satisfactory. ${ }^{3}$ Patients in an advanced stage of the disease however were also included and no data on the lesion size were provided.

The invention of monoclonal antibodies (MoAbs) by Köhler and Milstein ${ }^{4}$ and the formation of MoAbs against tumour-associated antigens added a new dimension to specific in vitro and in vivo tumour diagnosis. For in vivo application MoAbs can be labelled with $\gamma$-emitting isotopes and used as tracers for immunoscintigraphic tumour detection. Recent publications demonstrated the clinical value of the new diagnostic method in numerous malignant and benign diseases - for example, colorectal carcinoma, ovarian carcinoma, cutaneous melanoma, infection, thrombosis, and human cardiac transplant rejection. ${ }^{5-10}$

Up to now there are no specific MoAbs available against choroidal melanoma. However immunohistological studies of Bomanji ${ }^{11}$ and Scheiffarth ${ }^{12}$ showed the binding of $\mathrm{MoAb}$ $225 \cdot 28 \mathrm{~S}$ raised against cutaneous melanoma in choroidal melanoma. This class $\operatorname{IgG}_{2} \alpha$ antibody recognises the high molecular weight-melanoma associated antigen (HMW-MAA), a membranebound glycoprotein which is present on most cutaneous melanomas and to a lower extent in skin naevi and skin carcinomas. ${ }^{13}$ First preliminary in vivo studies by Bomanji ${ }^{14}$ and Scheidhauer ${ }^{15}$ were promising, but also emphasised the importance of further studies to evaluate the sensitivity and in particular the specificity of immunoscintigraphy.

This study was performed to define the value of immunoscintigraphy (IS) using the $\mathrm{MoAb}$ $225 \cdot 28 \mathrm{~S}$ for the diagnosis of choroidal melanoma in comparison with established diagnostic modalities and to determine the minimum lesion size for reliable imaging.

\section{Patients and methods}

A total of 101 patients with suspected choroidal melanoma were examined by IS after written consent had been obtained. Patients with a history of allergic reactions were excluded. Prior to the intravenous administration of the radiolabelled antibodies, the thyroid gland was blocked with perchlorate (50 drops). In most cases diagnosis was verified by ophthalmoscopy, ultrasound echography, and fluorescein angiography. Clinical follow-up in all non-melanoma lesions lasted more than 1 year. Ultrasound echography was performed according to the method described by Ossoinig' using the A- and B-image. Ten patients underwent enucleation because of the large tumour size.

The MoAb $225 \cdot 28 \mathrm{~S}\left(\mathrm{~F}\left(\mathrm{ab}^{\prime}\right)_{2}\right.$ fragments), directed against the HMW-MAA was delivered as a ready-to-use kit (Technemab Kl; Sorin 
Biomedica, Italy). Approximately $350 \mu \mathrm{g}$ of lyophilised antibodies were reconstituted by sterile insertion of $2 \mathrm{ml}$ sodium ${ }^{99 \mathrm{~m}} \mathrm{Tc}$ pertechnetate solution into the vial. Activity

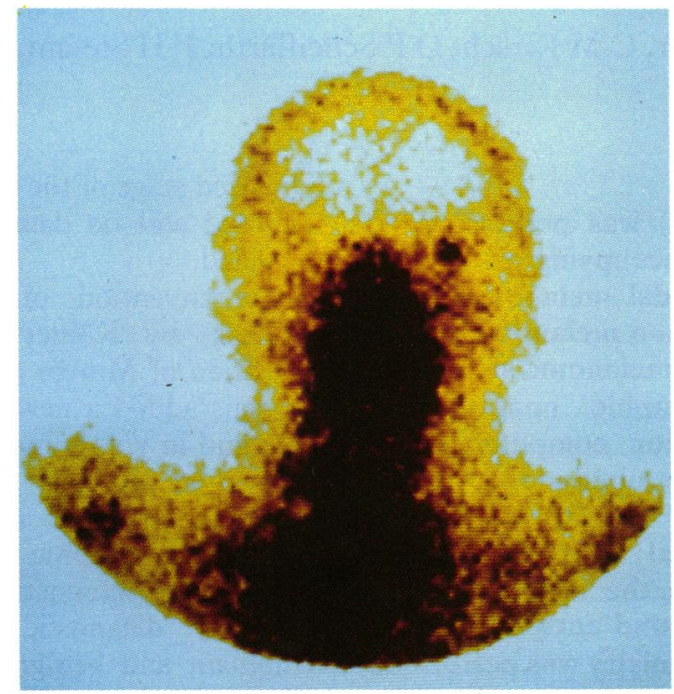

Fig $1 A$

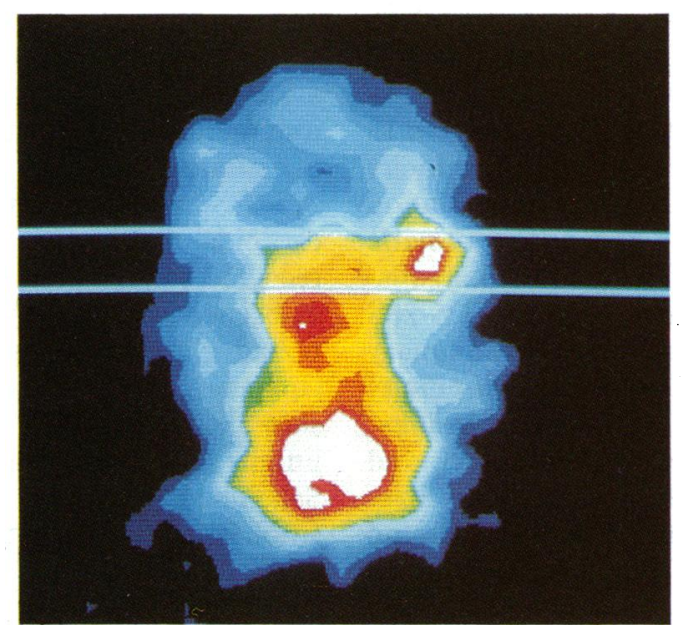

Fig $1 B$

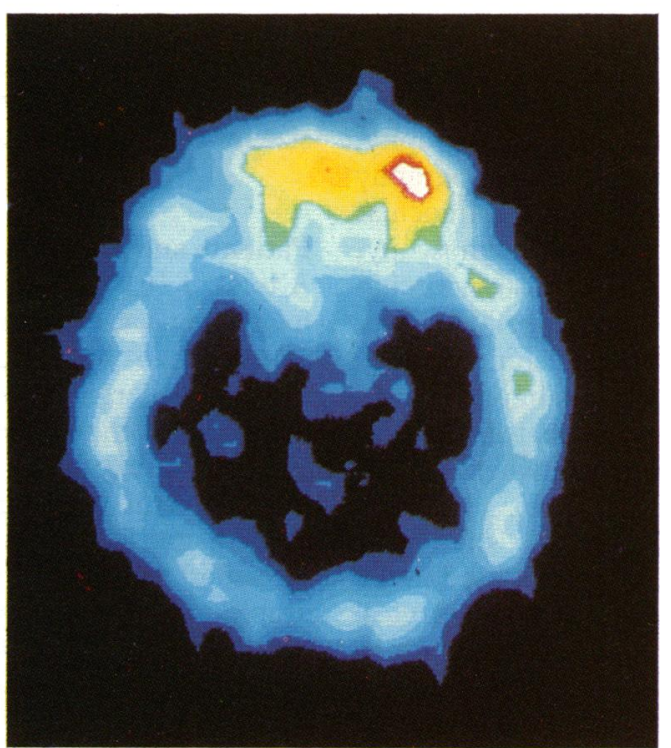

Fig $1 C$

Figure 1 A 69-year-old female patient. Antibody accumulation in a choroidal melanoma of the left eye, thickness $5 \cdot 7 \mathrm{~mm}$. Conventional imaging $(A)$ and SPECT images, frontal $(B)$ and transverse sections $(C)$.

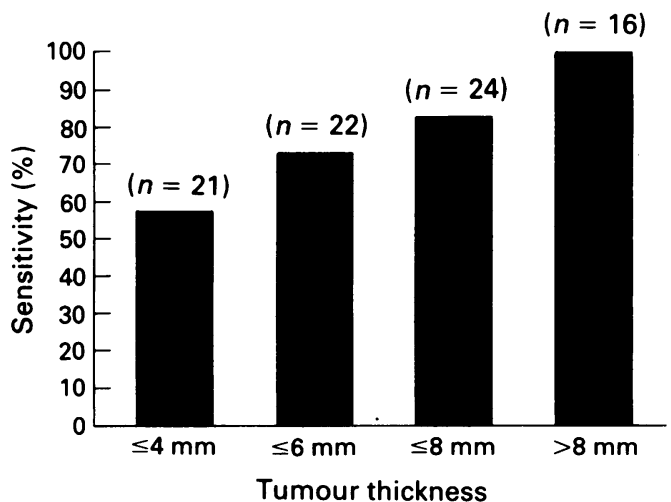

Figure 2 Correlation between thickness of choroidal melanomas, measured by ultrasonography and sensitivity of immunoimaging.

ranged between $700-1250 \mathrm{MBq}$ according to the time of imaging. After 15 minutes of incubation at room temperature, free ${ }^{99 \mathrm{~m}} \mathrm{Tc}$-pertechnetate was separated with a DEAE-Sephadex A25 ion exchange chromatography column. Labelling yield was $70-80 \%$. Then $450-1000 \mathrm{MBq}$ of radiolabelled antibody were diluted with isotonic $\mathrm{NaCl}$ solution to a total volume of $5 \mathrm{ml}$ and slowly injected intravenously.

For imaging the patient was placed in a supine position with the head fixed in a foam support with a caudal tilt of $15-30^{\circ}$. This position was chosen to avoid attenuation effects of the cranium and interference with non-specific activity uptake in the nasopharynx. Imaging was carried out 6 or 16 hours after antibody administration for practical reasons and from previous experience in cutaneous melanoma. ${ }^{1617}$ Static images were obtained with a large field of view gamma camera equipped with a high resolution collimator designed for low energies. Acquisition time was 15 minutes. A $128 \times 128$ computer matrix was utilised for data processing. Additionally, in all patients single photon emission computer tomography (SPECT) was performed. Either a single head (Gamma-tome, CGR) or a double-head (Rota, Siemens) rotating gamma camera system equipped with a high resolution collimator (parallel hole, $140 \mathrm{keV}$ ) was used. For single head acquisition images were acquired by continuous rotation over $360^{\circ}$ in 40 minutes and for the double head system by rotation in step and shoot mode $\left(360^{\circ}, 60\right.$ angular projections, 25 second acquisition/projection) around the patient. A total of at least 300000 counts for study in a $64 \times 64$ matrix were collected. Transverse sections were reconstructed by a filtered back-projection algorithm (SheepLogan-Hanning filter, cut-off frequency at $0 \cdot 5$, no attenuation correction) with subsequent computation of coronal and sagittal slices. Images were interpreted prospectively in a blind fashion without knowledge of the results of ophthalmoscopy and ultrasound echography and with no clinical information other than an instruction to rule out melanoma.

Cryostat sections of four out of 10 enucleated melanomas were investigated immunohistochemically to demonstrate MoAb binding. After incubation with anti-melanoma MoAb 225.28S (dilution 1:20) and second rabbit anti-mouse antibody sections were stained in alkaline-antialkaline phosphatase (APAAP). ${ }^{18}$ 


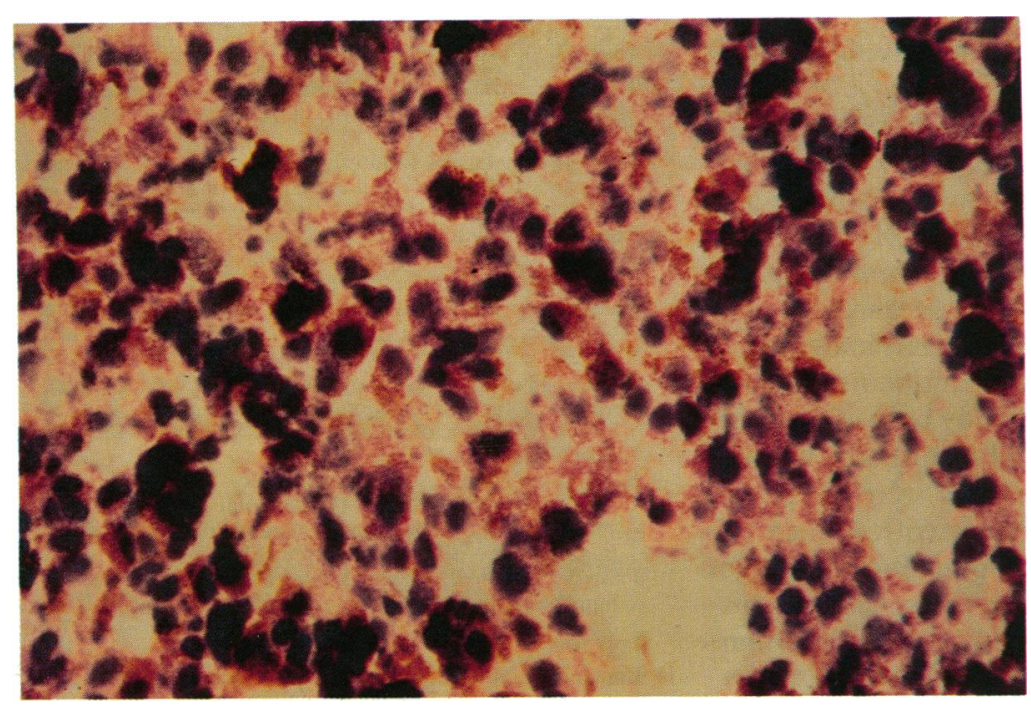

Figure 3 Immunohistochemistry with $M o A b$ $225 \cdot 28 S$ on choroidal melanoma section (APAAP reaction, dilution 1:20) counterstained with Mayer's haematoxylin. Magenta cytoplasmal staining indicates MoAb binding to $H M W-M A A$

Counterstaining is responsible for black colour reactions of nuclei.

Space occupying intraocular lesions investigated by IS included choroidal melanomas (85), naevi (11), non-melanoma metastases (three), and post-traumatic subretinal haemorrhages (two). Maximum lesion thickness measured by ultrasound echography ranged from $0 \mathrm{~mm}$ (not detectable) to $23 \mathrm{~mm}$ (mean $5.4 \mathrm{~mm}$ ). No clinical signs of metastases were observed in all the

\section{Results} melanoma patients. In 10 patients the melanoma-containing eye was enucleated with the clinical diagnosis confirmed by histopathology. All other patients were treated by irradiation with a ruthenium-106 scleral applicator.

No side effects or adverse reactions were noticed after the application of the antibody. In this study no data could be collected on the induction of human anti-mouse antibodies (HAMA) because there was no repeated antibody administration. However none of our patients with cutaneous melanoma who underwent repeated administration of the same MoAb under similar conditions had HAMA.

Sixty six out of 85 melanomas were visualised by the SPECT imaging technique (sensitivity $78 \%$ ). However only 35 of them were also detected by planar imaging. This results in a gain of the SPECT technique of $47 \%$. As expected in this stage of disease no brain metastases were diagnosed by IS. Non-specific tracer uptake was visualised in the nasopharynx, liver, kidneys, and bone marrow (Fig 1).

Detectability has shown a positive correlation with the thickness of the lesions measured by ultrasound echography: $\leq 4 \mathrm{~mm} 57 \%, \leq 6 \mathrm{~mm}$ $73 \%, \leq 8 \mathrm{~mm} 83 \%$, and $>8 \mathrm{~mm} 100 \%$ of choroidal melanomas were positive in IS. Mela-

Table 1 Results of immunohistochemistry $(I H)$ with the $M o A b 225 \cdot 28 S$ (APAAP method) compared to immunoscintigraphy (IS) with the same radiolabelled $M o A b$. Immunohistochemistry was possible on four out of 10 enucleated melanomas only

\begin{tabular}{lclcc}
\hline & & \multicolumn{3}{c}{ IS } \\
Patient & Lesion size $(\mathrm{mm})$ & planar & SPECT & IH \\
\hline M.R. & 8.9 & + & + & + \\
J.R. & $12 \cdot 5$ & + & + & + \\
B.A. & $8 \cdot 0$ & - & - & + \\
L.I. & $10 \cdot 8$ & + & + & + \\
\hline
\end{tabular}

nomas with a thickness below $2.7 \mathrm{~mm}(n=2)$ were not detected. Imaging was performed 6 or 16 hours following antibody application. Analysis of results revealed a higher sensitivity for early compared with delayed imaging, but this fact can be attributed to a different tumour size $(7.6 \mathrm{~mm}$ vs $6.1 \mathrm{~mm})$. No significant difference in sensitivity was seen between melanomas of nasal or temporal location: $86 \%$ of nasal (mean thickness $6.9 \mathrm{~mm}$ ) and $81 \%$ of temporal located melanomas (mean thickness $5.7 \mathrm{~mm}$ ) were visualised (Fig 2).

Fifteen of 16 non-melanoma lesions were truly negative (specificity 94\%). A slight accumulation of radioactivity in both eyes was seen in one patient with bilateral naevi in the SPECT slices only. This accumulation was not considered to be a positive result. All other naevi and the nonmelanoma metastases were negative. One false positive result was obtained in an old subretinal post-traumatic haemorrhage simulating a tumour mass (thickness $7 \cdot 4 \mathrm{~mm}$ ). IS could demonstrate the HMW-MAA in all sections examined (Fig 3 and Table 1).

\section{Discussion}

Our data show that lesions of a size even below an order of magnitude of resolution can be visualised. Thus the rate of antibody uptake seems to be the decisive parameter for tumour detection by IS. According to a recent study ${ }^{12}$ the expression of HMW-MAA was reported in all melanoma sections examined by immunohistochemistry though choroidal melanoma exhibited antigenic heterogeneity. ${ }^{19}$ Considering the small number of melanomas tested in all immunohistological studies, this suggested heterogeneity can be responsible for a sensitivity of IS below $100 \%$.

Conventional planar scintigraphy proved not to be sufficient for immunoscintigraphic imaging of choroidal melanomas. Taking into account the small size of melanomas, SPECT, the most advanced imaging technique in nuclear medicine, was used additionally and compared with conventional scintigraphy. SPECT images are created by a technique similar to $x$ ray computer tomography, which offers the selection of defined sections in transversal, frontal, and sagittal projection. Compared with planar scintigraphy SPECT provides equal at-best resolution, improved contrast without superimposed background activity, and better three-dimensional orientation. However, even with the SPECT imaging technique intraocular melanomas smaller than $2.7 \mathrm{~mm}$ in size were invisible.

Although brain metastases can be demonstrated by IS, non-specific activity accumulation makes the exclusion of liver metastases impossible. Occurrence of non-specific tracer uptake in liver, nasopharynx, and bone marrow is due to the well-known transchelation of DTPA-bound ${ }^{99 m} \mathrm{Tc}$ to transferrin and its accumulation in the reticuloendothelial system. Activity accumulation in the kidneys representing the physiological route for the excretion of antibody fragments $\left(F\left(a b^{\prime}\right)_{2}\right)$. Surprisingly, there was no significant difference in sensitivity between melanomas of nasal or temporal location as the 
non-specific nasopharyngeal uptake did not interfere with the detection of nasally located lesions.

All tumour markers used at present are not specific for a certain tumour, they are just expressed in a higher quantity by malignancies compared with normal tissue. The HMW-MAA is also present in skin naevi. Up to now there are no immunohistological data regarding the presence of this antigen in choroidal naevi. Normal eyes do not react with antibodies against the HMW-MAA. ${ }^{12}$ In our study only one out of eight naevi revealed a slight antibody uptake while all intraocular metastases were negative. These results are in agreement with Bomanji, ${ }^{20}$ who reported antibody accumulation in a large flat choroidal naevus. The presence of this antigen may indicate malignant transformation of the naevus. In the 2 year follow-up of this patient no clinical signs for tumour progression were encountered. Intensive activity accumulation was visualised in a second non-melanoma lesion, a subretinal haemorrhage following trauma. Haemorrhages can possess a higher blood pool which may be a possible explanation for this false positive result.

In conclusion, IS with MoAbs against the HMW-MAA proved to be a valuable diagnostic method which offers substantial sensitivity and specificity for the differentiation of intraocular space occupying lesions, particularly choroidal melanoma, naevus, and metastasis. It may add valuable information when the diagnosis cannot be confirmed by other diagnostic methods. Prospects for the future are the development of MoAbs against tumour antigens specific for choroidal melanoma ${ }^{21}$ as well as the injection of antibody cocktails against different epitopes, ${ }^{22}$ or the application of melanoma-specific haptens ${ }^{23}$ to improve immunoimaging of choroidal melanoma.

\section{In memory of Dr O F Scheiffarth.}

1 Ossoinig KC. Standard echography: basic principles, clinica applications and results. In: Dallow RL, ed. Ophthalmic ultrasonography: comparative techniques. Int Ophthalmol Clin 19/4. Boston: Little Brown, 1979: 127-210.

2 Shields JA. Accuracy and limitations of the ${ }^{32} \mathrm{P}$ test in the diagnosis of ocular melanoma: an analysis of 500 cases. diagnosis of ocular melanoma:
Ophthalmology 1978; 85: 950-66.
3 Liniecki J, Pertynski T, Krawczykowa Z, Stepien J, Durski $\mathrm{K} .{ }^{\mathrm{s}} \mathrm{Cr}$-bleomycin in the diagnosis of ocular melanoma. Nucl Med 1983; 22: 306-8.

4 Köhler G, Milstein C. Continuous cultures of fused cells secreting antibodies of predetermined specificity. Nature 1975; 256: 495-7.

5 Mach JP, Chatal JF, Lumbroso JD, et al. Tumor localization in patients by radiolabelled monoclonal antibodies against colon carcinoma. Cancer Res 1983; 43: 5593-600.

6 Chatal JF, Fumoleau P, Saccavini JC, et al. Immunoscintigraphy of recurrences of gynecologic carcinomas. $\mathcal{F}$ Nucl Med 1987; 28: 1807-19.

7 Buraggi GL. Radioimmunodetection of malignant melanoma with the $225 \cdot 28 \mathrm{~S}$ monoclonal antibody to HMW-MAA Nucl Med 1986; 25: 220-4.

8 Locher JT, Seybold K, Andres RY, et al. Imaging of inflammatory and infectious lesions after injection of radioiodinated monoclonal anti-granulocytes antibodies. Nucl Med Comm 1986; 7: 659-70.

9 Rosebrough SF, Grossman ZD, McAfee JG, et al. Thrombus localization with indium-111 and iodine-131 labelled fibrinspecific monoclonal antibody and its $F\left(a^{\prime}\right)_{2}$ - and $F a b-$ fragments. F Nucl Med 1988 29: 1212-22.

10 Frist W, Yasuda T, Segall G, et al. Noninvasive detection of human cardiac transplant rejection with In-111-labelled monoclonal antimyosin (Fab) imaging. Circulation 1987; 76: 81-5.

11 Bomanji J, Garner A, Prasad J, et al. Characterization of ocular melanoma with cutaneous melanoma antibodies. $\mathrm{Br} \mathcal{F}$ Ophthalmol 1987; 71: 647-50.

12 Scheiffarth OF, Strobl J, Stefani FH, et al. Tumormarke beim malignen Aderhautmelanom. Fortschr Ophthalmol beim malignen

13 Wilson BS, Jami K, Natali PG, Ferrone S. Distribution and molecular characterization of cell-surface and cytoplasmic antigen detectable in human melanoma cells with monoclonal antibodies. Int $\mathcal{F}$ Cancer 1981; 28: 293-300.

14 Bomanji J, Hungerford JL, Granowska M, Britton KE Radioimmunoscintigraphy of ocular melanoma with ${ }^{9 \%} \mathrm{~T}$ labelled cutaneous melanoma antibody fragments. $\mathrm{Br} \mathcal{F}$ Ophthalmol 1987; 71: 651-58.

15 Scheidhauer K, Markl A, Leinsinger G, et al. Immunoscintigraphy in intra-ocular malignant melanoma. Nucl Med Comm 1988; 9: 669-79.

16 Scheidler J, Leinsinger $G$, Landthaler $M$, Kirsch $C M$ Immunoscintigraphy in the follow-up of cutaneous Immunoscintigraphy in the follow-

17 Siccardi AG, Buraggi GL, Callegaro L, et al. A multicenter study of immunoscintigraphy with monoclonal antibodies in patients with melanoma. Cancer Res 1986; 46: 4817-22.

18 Cordell JL, Falini B, Erber WN, et al. Immunoenzymatic labelling of monoclonal antibodies using immune complexes of alkaline phosphatase and monoclonal anti-alkaline phosphatase (APAAP) complexes. $\mathcal{f}$ Histochem Cytochem 1984; 32: 219-29.

19 Van der Pol JP, Jager MJ, De Wolff-Rouendaal D, Ringens PJ, Vennegoor C, Ruiter DJ. Heterogeneous expression of melanoma-associated antigens in uveal melanomas. Curr Eye Res 1987; 6: 757-65.

20 Bomanii J Hungerford JL Granowska M, Britton KE Uptake of ${ }^{9 m_{\mathrm{m}} \mathrm{T}} \mathrm{c}$ labelled $\mathrm{F}\left(\mathrm{ab}^{\prime}\right)_{2}$ fragments of monoclonal antibody $225 \cdot 28 \mathrm{~S}$ by a benign ocular naevus. Eurf Nucl Med 1988; 14: 165-6.

21 Damato BE, Campbell AM, McGuire BJ, et al. Monoclonal antibodies to uveal melanoma. Eye 1987; 1: 686-90.

22 Matzku S, Kirchgessner H, Schmid U, et al. Melanoma targeting with a cocktail of monoclonal antibodies to distinct determinants of the human HMW-MAA. $\mathcal{F ~ N u c l ~ M e d ~} 1989$ 30: 390-7.

23 Le Doussal JM, Martin M, Gautherot E, et al. In vitro and in vivo targeting of radiolabelled monovalent and divalent haptens with dual specificity monoclonal antibody conjugates: enhanced divalent hapten affinity for cell-bound antibody conjugate. $\mathcal{F}$ Nucl Med 1989; 30: 1358-66. 